\title{
Downregulation of FEM1C enhances metastasis and proliferation in colorectal cancer
}

\author{
Runqing Huang ${ }^{1,2,3}$, Jianxia $\mathrm{Li}^{1,2}$, Yang Fu ${ }^{1,2}$, Yanhong Deng ${ }^{1,2,3}$ \\ ${ }^{1}$ Department of Medical Oncology, The Sixth Affiliated Hospital, Sun Yat-sen University, Guangzhou, China; ${ }^{2}$ Guangdong Research Institute of \\ Gastroenterology, The Sixth Affiliated Hospital, Sun Yat-sen University, Guangzhou, China; ${ }^{3}$ Guangdong Provincial Key Laboratory of Colorectal \\ and Pelvic Floor Diseases, Sixth Affiliated Hospital, Sun Yat-sen University, Guangzhou, China \\ Contributions: (I) Conception and design: R Huang; (II) Administrative support: Y Deng; (III) Provision of study materials or patients: Y Deng; \\ (IV) Collection and assembly of data: Y Fu; (V) Data analysis and interpretation: J Li; (VI) Manuscript writing: All authors; (VII) Final approval of \\ manuscript: All authors. \\ Correspondence to: Yanhong Deng. Department of Medical Oncology, The Sixth Affiliated Hospital, Guangdong Provincial Key Laboratory of \\ Colorectal and Pelvic Floor Diseases, Sun Yat-sen University, Guangzhou 510655, China. Email: dengyanh@mail.sysu.edu.cn.
}

Background: Feminization-1 (FEM-1) is considered a substrate recognition subunit of CUL2-RING E3 ubiquitin ligase complexes, which refers to sex determination by modulating TRA-1 stability in C. elegans. The function of mammalian orthologous gene of FEM-1 remains to be elucidated.

Methods: The expression of FEM1C in colorectal cancer (CRC) cells was interfered by small interference RNA (siRNA) transfection, and Cell counting kit-8 (CCK-8) assay, colony formation assay and transwell assay were performed. In order to estimate the function on metastasis, stable knockdown FEM1C cells were used to established liver and lung metastasis models. In addition, the expression of FEM1C in normal tissues, adenomas and tumor tissues were analyzed, and the relationship between FEM1C expression level and prognosis was analyzed by Kaplan-Meier method.

Results: Here, we report that the elimination of FEM1C, one of the members of FEM-1, significantly promoted the migration and invasion of colorectal cancer (CRC) cells in vitro and promoted liver and lung metastases in vivo. It also showed that the removal of FEM1C improved the proliferation ability of CRC cells. In particular, the cell shape changed from epithelial to fibroblast-like morphology. The tight cell monolayer was transformed into a dispersed distribution. Furthermore, it was demonstrated that FEM1C is down-regulated in tissues of CRC compared to normal tissues, and the high expression of FEM1C positively correlates with a good prognosis in patients with CRC. GSEA analysis showed that EMT signatures was enriched in FEM1C knockdown groups.

Conclusions: Down-regulation of FEM1C promotes proliferation and metastasis, and FEM1C may be a tumor suppressor in the development of CRC.

Keywords: FEM1C; colorectal cancer (CRC); metastasis; tumor suppressor

Submitted Aug 05, 2021. Accepted for publication Aug 30, 2021.

doi: 10.21037/atm-21-4244

View this article at: https://dx.doi.org/10.21037/atm-21-4244

\section{Introduction}

Colorectal cancer (CRC) is one of the most common malignant tumors and the most common cause of cancerassociated death worldwide. Although abnormal alteration of tumor suppressor and oncogene genes has been reported to drive CRC tumorigenesis and development (1), the precise molecular mechanisms of CRC pathogenesis, especially for distant metastases, remain elusive. A better understanding of the molecular mechanism driving metastasis and abnormal proliferation holds the promise of developing therapeutic strategies to eradicate CRC. 
Feminization-1 (FEM-1) is considered a substrate recognition subunit of CUL2-RING E3 ubiquitin ligase complexes, which were reported to participate in the determination of sex by modulating the stability of TRA-1 in C. elegans (2). The FEM1 protein participates in the assembly of E3 complexes by three elements, mainly, including the ANK repeat domains at the $\mathrm{N}$-terminus, which interact with substrates $(3,4)$, the $\mathrm{BC}$ box referring to binding of Elongin $\mathrm{B}$ and Elongin $\mathrm{C}$, and the cullin box referring to binding of cullin2 $(5,6)$. The mammalian orthologous gene consists of three members, including FEM1A, FEM1B, and FEM1C. Recently, two research groups have elucidated the molecular basis for substrate recognition by CUL ${ }^{\mathrm{FEM} 1} \mathrm{E} 3$ ligase by the crystallography method simultaneously, and it was shown that FEM1A, FEM1B, and FEM1C selectively targeted different classes of $\mathrm{Arg} / \mathrm{C}$-degrons $(7,8)$. However, we know little about the function of the FEM-1 gene in mammals, and few substrates of FEM-1 have been reported so far (9). In this study, we investigate the function of FEM1C in the development of CRC, because FEM1C is significantly down-regulated in CRC tissues, and low expression is related to the poor prognosis of CRC patients. We found that the elimination of FEM1C in CRC cell lines significantly promoted metastasis, both in vitro and in vivo. In addition, the removal of FEM1C also increased cell proliferation. The role of FEM1C in modulating cancer progression has not been reported before, and exploring the molecular function of it will extend our understanding of CRC pathogenesis and develop new therapeutic target.

Furthermore, an obvious morphology change was observed after the elimination of FEM1C in HCT116 and LoVo cells. Tandem mass tag (TMT) proteomic analysis revealed that the removal of FEM1C resulted in a change in the protein level of many proteins, which refers to cell adhesion, hypoxia, myogenesis, and epithelial-mesenchymal transition (EMT). Our study indicated that FEM1C might be a tumor suppressor and that its down-regulation may be an important factor in CRC development. We present the following article in accordance with the ARRIVE reporting checklist (available at https://dx.doi.org/10.21037/atm-21-4244).

\section{Methods}

\section{Cell lines}

Human embryonic kidney cell line HEK293T, human CRC cell lines (HCT116 and LoVo) were acquired from
ATCC and cultured in Dulbecco's modified Eagle's medium (DMEM) or RPMI 1640 medium supplemented with $10 \%(\mathrm{v} / \mathrm{v})$ fetal bovine serum (FBS) (Gibco), penicillin $(100 \mathrm{U} / \mathrm{mL})$, and streptomycin $(100 \mu \mathrm{g} / \mathrm{m} ;)$ at $37^{\circ} \mathrm{C}$ in a humidified incubator with $5 \% \mathrm{CO}_{2}$, respectively.

\section{RNA extraction and $q R T-P C R$}

Total RNA was extracted using the Total RNA Kit I (Omega) according to the manufacturer's protocol and reverse transcribed into cDNA with the EasyScript one-step gDNA removal and cDNA synthesis SuperMix (TransGen Biotech). Real-time PCR was performed using TransStart ${ }^{\circledR}$ Tip Green qPCR SuperMix (TransGen Biotech). The PCR procedure was described as follows: $95{ }^{\circ} \mathrm{C}$ for $3 \mathrm{~min} ; 95^{\circ} \mathrm{C}$ for $15 \mathrm{sec}, 60^{\circ} \mathrm{C}$ for $30 \mathrm{sec}, 72^{\circ} \mathrm{C}$ for $15 \mathrm{sec}(45$ cycles). The primers used for qRT-PCR are shown in Table S1. The $2^{-\Delta \Delta \mathrm{Ct}}$ method determined the relative gene expression levels, with GAPDH as the internal control.

\section{RNA interference and transwell assay}

Cells were seeded in a $6 \mathrm{~cm}$ diameter Petri dish with $50-60 \%$ confluence, and siRNA was transfected into cells at final concentrations of $100 \mathrm{nM}$. The stable knockdown cell line was established by infecting HCT116 with lentiviruses carrying shRNA expression cassette, followed by a one-week selection of $0.5 \mu \mathrm{g} / \mathrm{mL}$ puromycin. Detailed information on siRNA and shRNA is shown in Table S1.

Cells were digested with $0.25 \%$ trypsin and suspended in an FBS-free medium. 24 well transwell plates with $8-\mu \mathrm{m}$ pore size polycarbonate filter (Corning) were used for the Transwell assay. $10^{5}$ cells in $200 \mu \mathrm{L}$ volume were seeded in the upper cell, and $600 \mu \mathrm{L}$ RPMI 1640 with $20 \%$ FBS was injected into the lower space. Cells were fixed with $4 \%$ paraformaldehyde and then stained with $1 \%$ crystal violet after 36 hrs. The upper cells of the filter were erased with a cotton swab, and after twice rinsing with water, the lower cells were photographed at $100 \times$.

\section{Cell counting kit-8 (CCK-8) assay and colony formation assay}

$5 \times 10^{3}$ cells were seeded into 96 -well flat-bottom plates, and cell proliferation was determined daily using the CCK-8 assay (Beyotime, Shanghai, China). The substrate was added of $5 \mu \mathrm{L}$ per well, and after $1 \mathrm{hr}$ of incubation, absorbance was measured at $450 \mathrm{~nm}$ on a plate reader (Thermo Fisher Scientific). 
Five hundred cells were seeded in 6-well culture plates and cultured for 10 days. Cells were fixed with $4 \%$ paraformaldehyde for $10 \mathrm{~min}$, followed by staining with $1 \%$ crystal violet for $15 \mathrm{~min}$. The plates were photographed, and the colonies were counted.

\section{Models of liver and lung metastasis in vivo}

Liver and lung metastasis experiments in nude mice were designed and performed to comply with the approved protocol described by the Sixth Affiliated Hospital of Sun Yat-sen University (20190821-006), in compliance with guidelines of The Sixth Affiliated Hospital of Sun Yat-sen University for the care and use of animals. A protocol was prepared before the study without registration. The Beijing Charles River Laboratories supplied male and female BALB/c-nude mice (3-4 weeks old). The liver metastasis models were established by injecting cancer cells into the spleen, and the lung metastasis models were established by injecting cancer cells into a caudal vein. Cells were suspended in PBS, and the injection number was $2 \times 10^{6}$ per mouse ( 5 mice per group). Five weeks later, livers and lungs were harvested and fixed with a $10 \%$ formalin solution. The metastasis nodules were counted. The animals were excluded if they died after surgery in one week because of failure of surgery.

\section{Immunofluorescent assay (IFA)}

Cells were washed twice with PBS, fixed with $4 \%$ paraformaldehyde for 20 minutes, then permeabilized with $0.2 \%$ Triton X-100 in PBS for 10 minutes followed by incubation with normal goat serum (Boster, China) for $1 \mathrm{hr}$ at room temperature. The cells were then incubated with a primary antibody for 2 hrs. After washing four times (5 min per time) with PBST (PBS containing $0.2 \%$ between 20), a secondary antibody conjugated 488 or $594 \mathrm{~nm}$ fluorophore (ThermoFisher) was added and incubated for $1 \mathrm{hr}$. The nuclei were stained with Hoechst 33342 (Thermo Fisher Scientific, USA). Images were captured with a Leica confocal microscope.

\section{Protein extraction and Western blot analysis}

Total protein was extracted using RIPA buffer with the protease inhibitor cocktail (Beyotime Biotechnology) on ice for $30 \mathrm{~min}$. Protein concentration was measured with the BCA Protein Assay Kit (ThermoFisher). Proteins were separated by SDS-PAGE and transferred to a nitrocellulose membrane (Millipore). After being blocked with $10 \%$ skim milk for $1 \mathrm{hr}$ at room temperature, membranes were incubated with specific primary antibodies at $4{ }^{\circ} \mathrm{C}$ overnight. After washing four times with PBST (PBS plus $0.5 \%$ Tween-20), membranes were incubated with secondary antibodies for $1 \mathrm{hr}$ at room temperature. After washing, protein bands were detected by enhanced chemiluminescence reagent (ThermoFisher).

\section{GEO and TCGA data analysis}

The data for the GSE8671, GSE32323, and GSE39582 transcriptome profiles were downloaded from GEO (Gene Expression Omnibus) (https://www.ncbi.nlm.nih.gov/ geo/), and the CRC gene expression profile published by TCGA (The Cancer Genome Atlas) was downloaded from OncoLnc (http://www.oncolnc.org/) and UALCAN (http://ualcan.path.uab.edu/index.html) website. Survival data of CRC patients in TCGA and GSE39582 were also downloaded together. The transcriptional level of FEM1C between CRC or adenoma tissues and normal tissues was compared. The Kaplan-Meier method acquired the survival curves (OS) and relapse-free survival (RFS) curves, and differences were analyzed with the log-rank test. X-tile statistical software determined the cut-off value between the high and low FEM1C expression groups (Version 3.6.1). The study was conducted in accordance with the Declaration of Helsinki (as revised in 2013).

\section{Proteomic analysis of tandem mass tags (TMT) $(10,11)$}

HCT116 was transfected with the negative siRNA or the siRNA-targeted FEM1C gene. 48 hrs later, SDT buffer was added and lysate was sonicated and then boiled for $15 \mathrm{~min}$. After centrifuged at $14,000 \mathrm{~g}$ for $40 \mathrm{~min}$, the supernatant was quantified with the BCA Protein Assay Kit (P0012, Beyotime). $200 \mu \mathrm{g}$ of proteins were incorporated into $30 \mu \mathrm{L}$ SDT buffers (150 mM Tris-HCl, 4\% SDS, 100 mM DTT, pH 8.0). DTT, Detergent and other low molecular weight components were removed by ultrafiltration (Sartorius, $30 \mathrm{kD}$ ) using UA buffer (8 M Urea, $150 \mathrm{mM}$ Tris-HCl $\mathrm{pH}$ 8.5). Following, $100 \mu \mathrm{L}$ iodoacetamide (100 mM IAA in UA buffer) was added to block reduced cysteine residues, and samples were incubated for $30 \mathrm{~min}$ in darkness. The filters were washed with $100 \mu \mathrm{L}$ UA buffer for three times and then with $100 \mu \mathrm{L} 0.1 \mathrm{M}$ TEAB buffer for twice. Finally, the protein suspensions were digested with $4 \mu \mathrm{g}$ trypsin 
(Promega) in $40 \mu \mathrm{L} 0.1 \mathrm{M}$ TEAB buffer overnight at $37^{\circ} \mathrm{C}$, and the resulting peptides were collected. The peptide content was determined by UV light spectral density at $280 \mathrm{~nm}$ using an extinction coefficient of 1.1 of $0.1 \%$ solution $(\mathrm{g} / \mathrm{L})$ calculated based on the frequency of tryptophan and tyrosine in vertebrates' proteins. According to the manufacturer's protocols, $100 \mu \mathrm{g}$ peptide mixture of each sample was labeled with TMT reagent (Thermo Fisher Scientific).

TMT-labeled peptides were fractionated by RP chromatography using the Agilent 1260 Infinity II HPLC. Subsequently, the peptides were analyzed using LC-MS/ MS on QExactiveTM Plus (Thermo Fish Scientific) coupled to an EASY-nLC 1000 UPLC system (Thermo Fisher Scientific). LC-MS/MS analysis was performed on a Q Exactive Plus mass spectrometer (Thermo Fisher Scientific) coupled to Easy nLC (Thermo Fisher Scientific) for $90 / 120 \mathrm{~min}$ (determined by project proposal). The mass spectrometer was operated in positive-ion mode. The MS data was dynamically acquired using a data-dependent top10 method, choosing the most abundant precursor ions from the survey scan $(350-1,800 \mathrm{~m} / \mathrm{z})$ for the fragmentation of HCD. The automatic gain control (AGC) target was set to $3 \mathrm{e} 6$, with a maximum inject time of $45 \mathrm{~ms}$. Survey scans were acquired at a resolution of 70,000 at $\mathrm{m} / \mathrm{z} 200$, and the resolution for HCD spectra was set at 17,500 at $\mathrm{m} / \mathrm{z} 200$, and the isolation width was $2 \mathrm{~m} / \mathrm{z}$. The normalized collision energy was $30 \mathrm{eV}$. MS/MS spectra were searched using the MASCOT engine (Matrix Science, London, UK; version 2.6) embedded in Proteome Discoverer 2.1. The following parameters were set. Proteins which fold change $>1.2$ and $\mathrm{P}$ value (student's $t$-test) were differentially expressed proteins.

All protein sequences were aligned with the Homo sapiens database downloaded from NCBI (NCBI-blast2.2.28+-win32.exe); only the sequences in the top 10 and E-value $\leq 1 \mathrm{e}-3$ were kept. A two-way ANOVA test was applied to detect differentially expressed proteins between FEM1C knockdown and control samples. The $\mathrm{P}$ values were corrected using the Benjamini-Hochberg procedure. Pathway enrichment analysis was executed using GSEA analysis based on the MSigDB database using the R/ Clusterprofiler package. The pathways were considered enriched in the specified group if the false discovery rate (q value) of the GSEA analysis was $<0.05$.

\section{Statistical analysis}

Data are expressed as mean \pm standard deviation. A two- sided unpaired Student's $t$-test was used to evaluate the statistical differences between two groups, unless otherwise stated. It was statistically significant when the $P$ value is less than 0.05 . The asterisks indicate statistical differences ${ }^{* * *}$, $\mathrm{P}<0.001$; **, $\left.\mathrm{P}<0.01 ;{ }^{*}, \mathrm{P}<0.05\right)$. Data analysis was performed using GraphPad Prism 5 software.

\section{Results}

\section{FEM1C is down-regulated in CRC, and its expression is} positive with a good prognosis in CRC patients

To evaluate the expression of FEM1C during the development of CRC, we analyzed the transcriptome profile data of normal tissues, adenomas, and CRC tissues. FEM1C was shown to be significantly down-regulated in adenomas and CRC tissues compared to normal tissues (Figure 1A,1B and Figure S1A). Comparable results were displayed by analyzing TCGA data (Figure S1B). Furthermore, it was demonstrated that patients with colon adenocarcinoma (COAD) and rectum adenocarcinoma (READ) with high expression of FEM1C had longer overall survival times and disease-free survival times (Figure 1C,1D and Figure S1C,S1D) using the Kaplan-Meier method with the logarithmic rank test. These results suggested that FEM1C may be positively correlated with the patient's prognosis and may be a tumor suppressor in CRC development.

\section{Knockdown of FEM1C promotes migration, invasion, and metastasis of CRC cells}

We have found that the knockdown of FEM1C by siRNA could promote migration and invasion of HCT116 cells in vitro (Figure $2 A$ ). In addition, an obvious change in cell shape was observed when FEM1C was knocked down (Figure $2 B$ and Figure S2A). Comparable results were obtained in LoVo cells (Figure S2B,S2C). The FEM1 gene contains three members, FEM1A, FEM1B, and FEM1C in Homosapiens. Interestingly, the elimination of FEM1A and FEM1B did not change the cell morphology (Figure S2D). It seems that FEM1C has the specific function to modulate cell morphology and mobility, which is absent in FEM1A and FEM1B. To investigate whether the knockdown of FEM1C can improve metastasis in vivo, we established liver and lung metastasis models by injecting CRC cells into the spleen and caudal vein. As the results have shown, the knockdown of FEM1C by shRNA significantly promoted the seeding of HCT116 in the liver and lung (Figure 2C,2D). All the above 

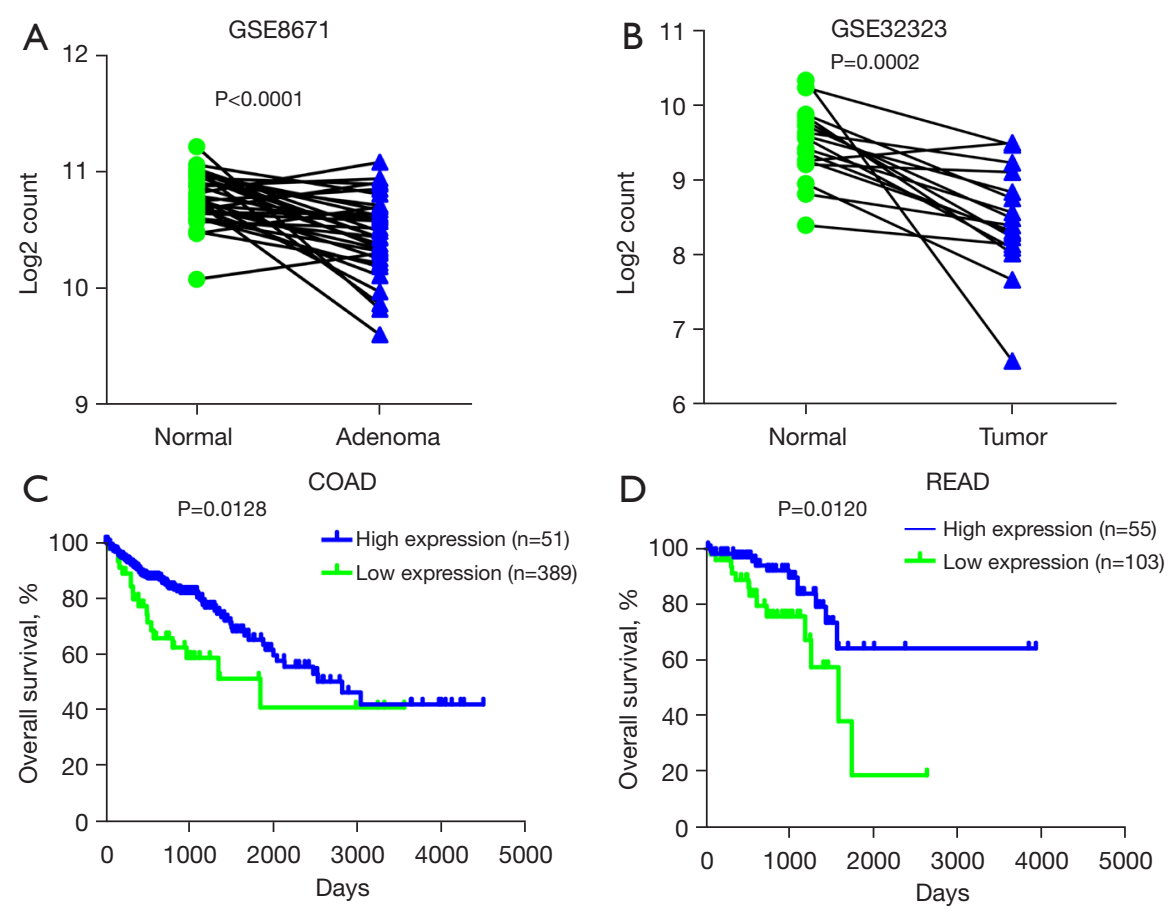

Figure $1 \mathrm{FEM1C}$ is down-regulated in adenomas and CRC tissues, and its expression level is correlated with the prognosis of patients with CRC. (A) The expression level of FEM1C in 32 pairs of adenomas and normal mucosa was analyzed. The data was from GSE8671. (B) The expression level of FEM1C in 17 pairs of cancer and normal tissues was analyzed. The data was from GSE32323. Two-side paired Student $t$ test was used to evaluate the statistical differences of two groups. (C,D) The relationship between FEM1C expression level and overall survival times in COAD (C) and READ (D) patients were analyzed by Kaplan-Meier method with Log-rank test. The data was downloaded from OncoLnc (http://www.oncolnc.org/). CRC, colorectal cancer.

evidences indicated that FEM1C knockdown promoted metastasis of CRC cells in vitro and in vivo.

\section{Knockdown of FEM1C promotes proliferation of CRC cells}

Abnormal proliferation is one of the malignant features of cancers. Therefore, we had studied the function of FEM1C in cell proliferation. The CCK-8 assay showed that HCT116 and LoVo cells reproduced faster when down-regulating FEM1C by siRNA (Figure $3 A$ and Figure S3A). The results were consistent in the colony formation assay; there were more colonies in the FEM1C knockdown groups than in the negative controls (Figure 3B and Figure S3B). These revealed that FEM1C participated in modulating the cell cycle, and its down-regulation promoted cell proliferation.

\section{Knockdown of FEM1C up-regulates proteins involved in the EMT}

To better understand the mechanisms by which FEM1C affects the malignant features of CRC, we performed a proteomic analysis of HCT116 transfected with negative control siRNA and the FEM1C targeted siRNA. A cutoff value of fold change $(\mathrm{FC})>1.5$ and adjusted $\mathrm{P}$ value $\leq 0.05$ was used, and there were 59 differentially expressed proteins (DEP) after FEM1C down-regulation (Figure 4A). Thirty-two proteins were significantly up-regulated in the FEM1C knockdown groups, while 27 proteins were significantly down-regulated. GSEA analysis showed that several gene signatures were enriched in FEM1C knockdown groups, including cell adhesion, hypoxia, myogenesis, and EMT (Figure 4B,4C) which can interpret the enhancement of malignant features when FEM1C was down-regulated. It is accepted that EMT plays a key role in tumor metastasis. The main core genes that contribute to the enrichment of the EMT pathway were significantly up-regulated in the FEM1C knockdown groups, including TGA2, NT5E, CALD1, FUCA1, and EMP3 (Figure 4D,4E). These results indicated that FEM1C negatively regulates the expression of EMT-associated 

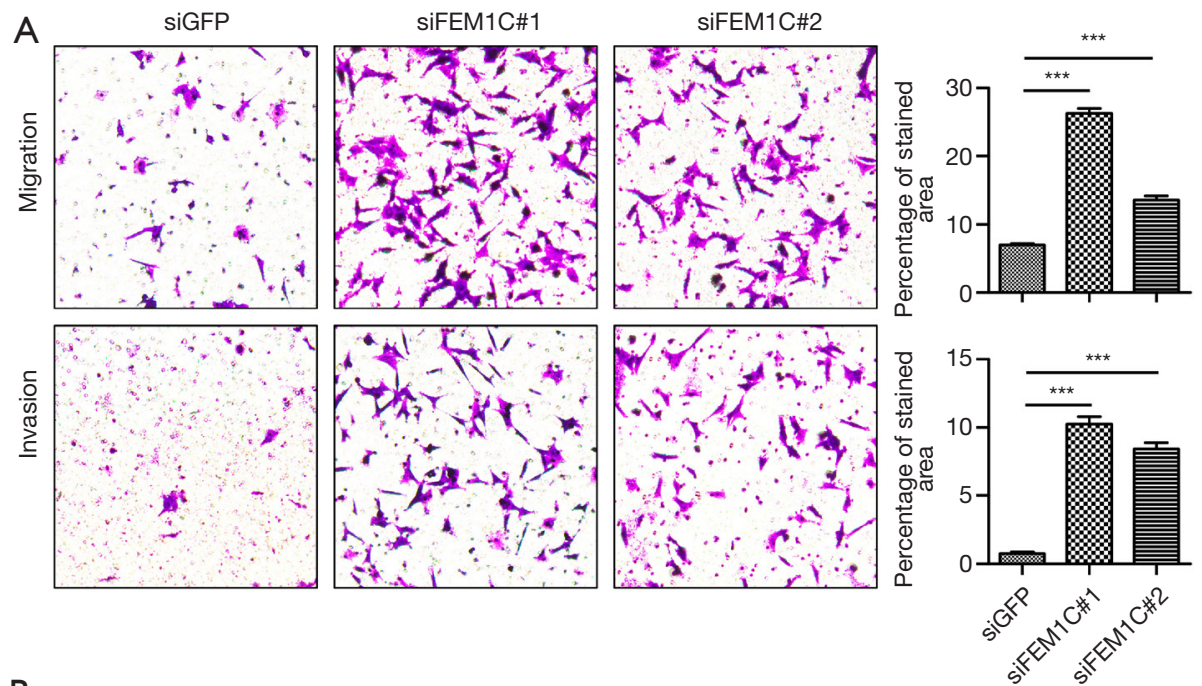

B
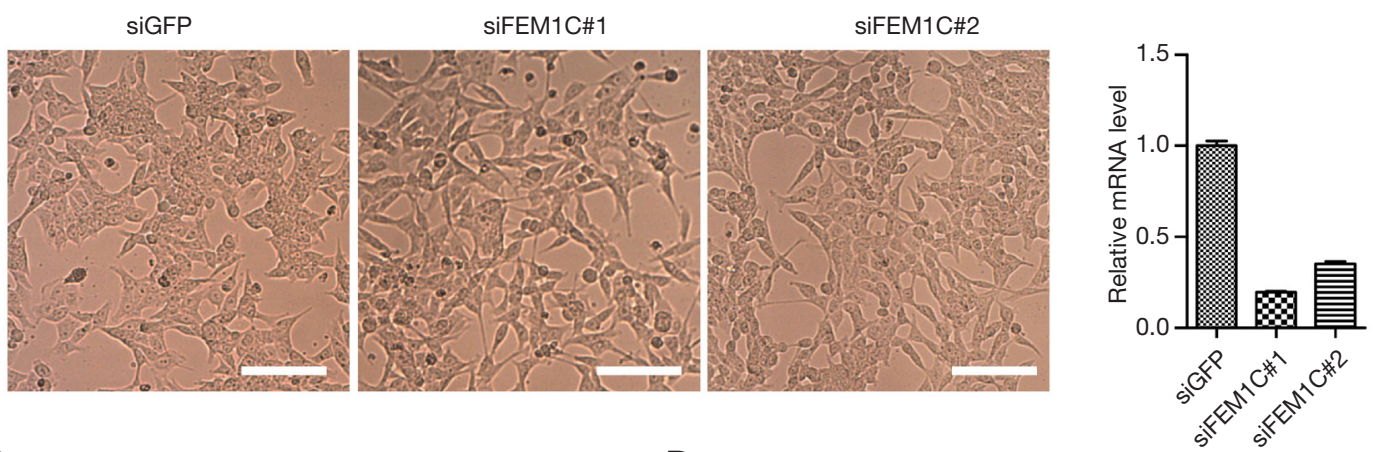

C

D
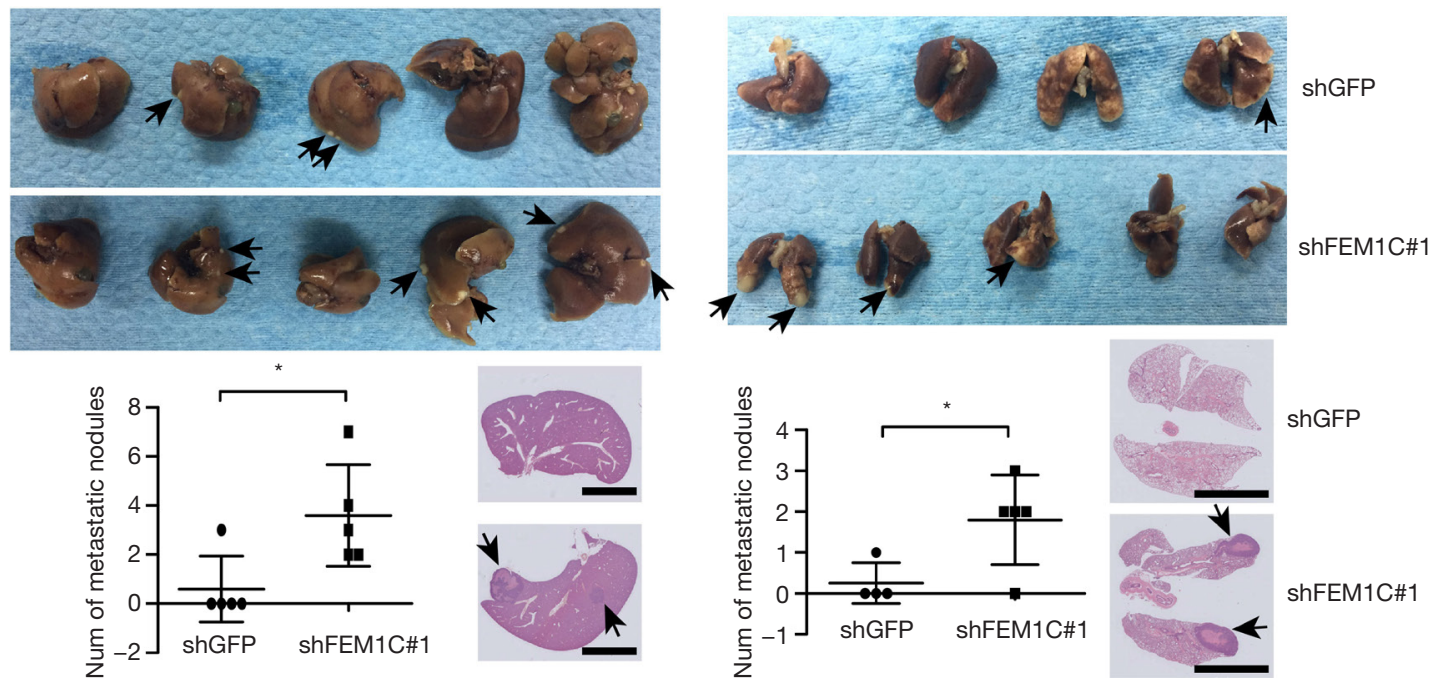

Figure 2 FEM1C knockdown promoted metastasis in CRC. (A) After transfection with siRNA for 48 hrs, cells were harvested to perform the transwell assay (magnification times, 100×; ${ }^{* * *}, \mathrm{P}<0.001$; * $\mathrm{P}<0.05$ ). (B) HCT116 cells were transfected with indicated siRNAs, and 48 hrs later, the cells were observed on microscopy and photographed (left). The right panel showed the knockdown efficiency of siRNAs. Scale bar $=100 \mu \mathrm{m}$. (C,D) Stable FEM1C knockdown cells were injected into the spleen and caudal vein. Five weeks later, the livers and lungs were harvested and fixed. The numbers of metastasis nodules were counted, and the histological chemistry method detected the nodules with HE staining (hematoxylin-eosin). Arrowhead indicated metastasis nodules in the liver and lung. Scale bar $=5 \mathrm{~mm}$. CRC, colorectal cancer. 

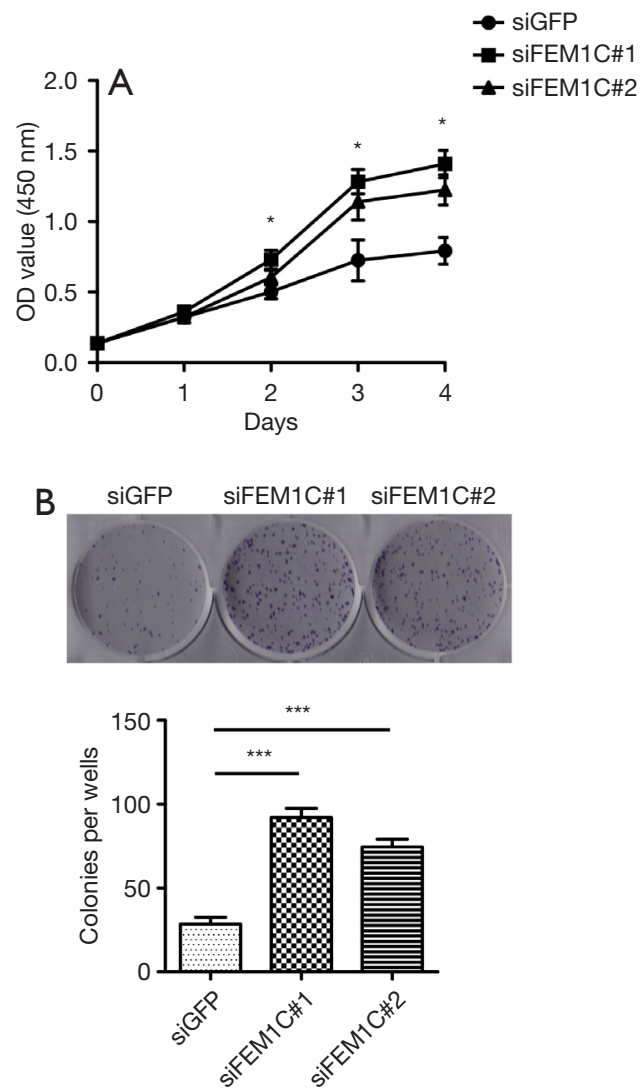

Figure 3 FEM1C knockdown promoted cell proliferation. HCT116 cells were transfected with indicated siRNAs, and cell proliferation was determined by the CCK- 8 assay $\left({ }^{*}, \mathrm{P}<0.05\right)$ (A) and the colony formation assay (***, $\mathrm{P}<0.001)(\mathrm{B})$. For colony formation assay, five hundred cells were seeded in 6-well culture plates and cultured for 10 days. Cells were fixed with $4 \%$ paraformaldehyde for $10 \mathrm{~min}$, followed by staining with $1 \%$ crystal violet for $15 \mathrm{~min}$. The plates were photographed, and the colonies were counted.

proteins that contribute to migration, invasion, and metastasis in CRC. It is noteworthy that conventional EMT markers, including vimentin and fibronectin, have no change in protein level when FEM1C was down-regulated (Figure S4A). Although E-cadherin was shown to be slightly up-regulated in proteomic data, no obvious upregulation was observed in the Western Blot assay (Figure S4B).

\section{Discussion}

In this study, we demonstrated that FEM1C knockdown promoted CRC cell proliferation and metastasis, both

in vitro and in vivo, and low expression of FEM1C was related to the poor prognosis of CRC patients, revealing the tumor suppressor role of FEM1C in CRC development. There were three members of the FEM1 gene characterized in mammals, FEM1A, FEM1B, and FEM1C (12). We observed obvious morphological changes after interfering with FEM1C in HCT116 and LoVo cells, but not after interfering with FEM1A and FEM1B, which may be attributed to selective targeting of substrates of different members of FEM1 $(7,8)$. Although FEM1C is considered the substrate recognition subunit in mammals, as invertebrates, few substrates have been identified, and its biological function in mammalian remains to be clarified $(13,14)$. FEM1C can also exert a function in mammalian cells independent of the assembly of the CUL2 ${ }^{\mathrm{FEM1C}} \mathrm{E} 3$ complex. It was supported that the knockdown of cullin2, the scaffold of the CUL2 ${ }^{\mathrm{FEM} 1 \mathrm{C}} \mathrm{E} 3$ complex, did not change the cell morphology and mobility (data not shown). Whether the E3 ligase independent function of FEM1C exists or not needs further investigation in the future.

EMT is a process known as the transdifferentiation of epithelial cells into mesenchymal cells during embryogenesis, wound healing, and cancer progression (15). It is widely considered that EMT plays a key role in tumor metastasis to acquire mobility to intravasation and extravasation (16). Proteomic analysis when FEM1C was down-regulated showed that differentially expressed proteins were enriched in the EMT pathway, and some proteins referring to EMT, including TGA2, NT5E, CALD1, FUCA1, and EMP3, were significantly up-regulated. EMT refers to the reprogramming of gene expression mediated by key transcription factors, including zinc finger E-box binding (ZEB), SNAI1 and basic helix loop transcription factors $(15,17)$. However, we found no expression of ZEB1, SNAI1, SLUG, TWIST, the key transcription factors of EMT in proteomic data (data not shown).

Furthermore, conventional EMT markers, including E-cadherin, vimentin, and fibronectin, did not change in protein levels when FEM1C was knocked down. This evidence suggests that the morphological change by eliminating FEM1C is not mediated by conventional mechanisms of EMT driven by several transcription factors. The detailed mechanisms need further study. The Rho/ ROCK/LIMK pathway regulates the dynamic of the actinnetwork, inhibiting cofilin by phosphorylating it on serine 3 residues. Cofilin is an actin depolymerization factor that facilitates actin cytoskeleton remodeling $(18,19)$. The Rho/ ROCK pathway inhibition was reported to result in the 


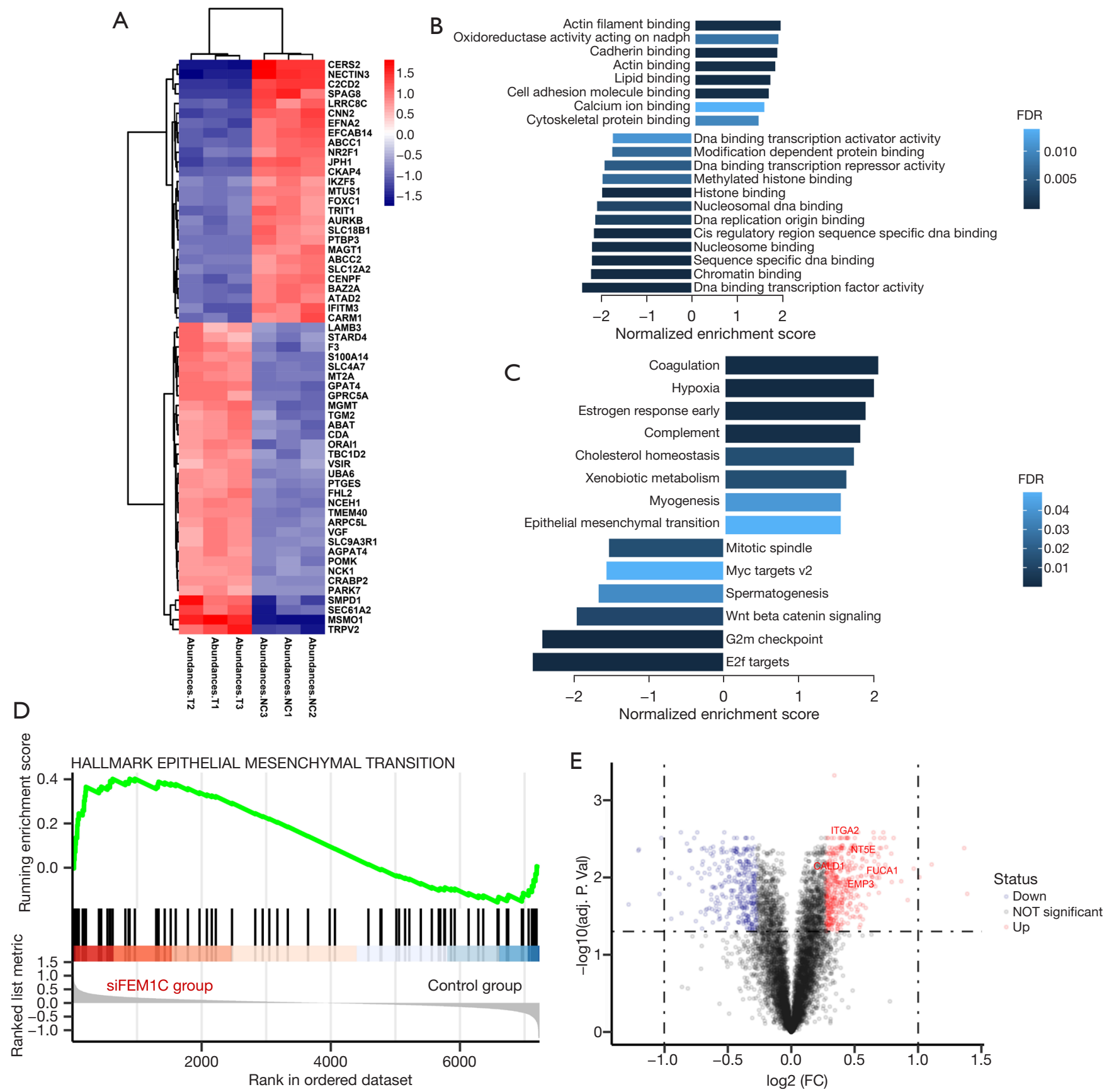

Figure 4 Proteomic analysis revealed changes in protein profile when FEM1C was downregulated. (A) Hierarchical clustering and heatmap representation of differentially expressed proteins between FEM1C knockdown groups and controls. (B) Bar graph representation of the top significantly enriched pathways in the FEM1C knockdown groups compared to controls identified by GSEA of the sets of molecular function genes of GO. (C) Bar plot representation of top significantly enriched pathways in the FEM1C knockdown group compared to the controls identified by GSEA of the set of hallmark genes. (D) Enrichment plot of epithelial-mesenchymal transition (EMT) genes in FEM1C knockdown groups compared to controls. (E) Volcano plot of differentially expressed proteins between FEM1C knockdown groups and controls in HCT116 cells. Significantly up-regulated and down-regulated proteins are highlighted in red and blue, respectively (FC $>1.5$; adjust $\mathrm{P}$ value $<0.05)$, among which proteins involved in EMT were labeled. EMT, epithelial-mesenchymal transition. 
loss of actin stress fibers, cell elongation, and increased cell motility $(20,21)$, as in our study. The next investigation will explore whether FEM1C-mediated morphological change and increased cell mobility result from the actin network dynamic change.

It was demonstrated that FEM1C knockdown promoted cell proliferation. In fact, by proteomic analysis, we also found the upregulation of CCND1 in FEM1C interfering cells and the downregulation of CDK2AP1 which is a specific inhibitor of the cell-cycle kinase (CDK2) (data not shown). CCND1 interacts and activates CDK4 which phosphorylates and inhibits the retinoblastoma (RB) proteins such as RB1. Phosphorylation of RB1 releases E2F from the RB/E2F complex and allows the transcription of E2F target genes which are driving the progression through the G1 phase (22). Elevated CCND1 promoted tumor progression and related to poor survival in several solid tumors (23). CDK2AP1 was considered as a tumor suppressor, and it was reported that RNAi-mediated CDK2AP1 inhibition was associated with decreased apoptosis and increased cell proliferation in CRC cell lines $(24,25)$. Downregulation of FEM1C may promote CRC proliferation by mediating the protein level of CCND1 and CDK2AP1.

In conclusion, our findings showed that downregulation of FME1C promotes CRC progression and relates with poor survival. Clarifying the detailed mechanisms of FEM1C-mediated cell mobility and proliferation would provide new insights into the progression of CRC.

\section{Acknowledgments}

Funding: This work was supported by the National Natural Science Foundation of China (81974369), the Guangdong Province Natural Science Foundation (2019A1515010071), the National Key Research and Development Program of China (2019YFC1316003).

\section{Footnote}

Reporting Checklist: The authors have completed the ARRIVE reporting checklist. Available at https://dx.doi. org/10.21037/atm-21-4244

Data Sharing Statement: Available at https://dx.doi. org/10.21037/atm-21-4244

Conflicts of Interest: All authors have completed the ICMJE uniform disclosure form (available at https://dx.doi. org/10.21037/atm-21-4244). The authors have no conflicts of interest to declare.

Ethical Statement: The authors are accountable for all aspects of the work in ensuring that questions related to the accuracy or integrity of any part of the work are appropriately investigated and resolved. All procedures were conducted in accordance with the Declaration of Helsinki (as revised in 2013). Animal experiments were performed under a project license (20190821-006) granted by ethics board of The Sixth Affiliated Hospital of Sun Yat-sen University, in compliance with guidelines of The Sixth Affiliated Hospital of Sun Yat-sen University for the care and use of animals.

Open Access Statement: This is an Open Access article distributed in accordance with the Creative Commons Attribution-NonCommercial-NoDerivs 4.0 International License (CC BY-NC-ND 4.0), which permits the noncommercial replication and distribution of the article with the strict proviso that no changes or edits are made and the original work is properly cited (including links to both the formal publication through the relevant DOI and the license). See: https://creativecommons.org/licenses/by-nc-nd/4.0/.

\section{References}

1. Leggett B, Whitehall V. Role of the serrated pathway in colorectal cancer pathogenesis. Gastroenterology 2010;138:2088-100.

2. Starostina NG, Lim JM, Schvarzstein M, et al. A CUL-2 ubiquitin ligase containing three FEM proteins degrades

TRA-1 to regulate C. elegans sex determination. Dev Cell 2007;13:127-39.

3. Wolf D, Hofbrucker-MacKenzie SA, Izadi M, et al. Ankyrin repeat-containing N-Ank proteins shape cellular membranes. Nat Cell Biol 2019;21:1191-205.

4. Spence AM, Coulson A, Hodgkin J. The product of fem-1, a nematode sex-determining gene, contains a motif found in cell cycle control proteins and receptors for cell-cell interactions. Cell 1990;60:981-90.

5. Mahrour N, Redwine WB, Florens L, et al. Characterization of Cullin-box sequences that direct recruitment of Cul2-Rbx1 and Cul5-Rbx2 modules to Elongin BC-based ubiquitin ligases. J Biol Chem 2008;283:8005-13.

6. Kamura T, Maenaka K, Kotoshiba S, et al. VHL-box and SOCS-box domains determine binding specificity for 
Cul2-Rbx1 and Cul5-Rbx2 modules of ubiquitin ligases. Genes Dev 2004;18:3055-65.

7. Chen X, Liao S, Makaros Y, et al. Molecular basis for arginine C-terminal degron recognition by Cul2FEM1 E3 ligase. Nat Chem Biol 2021;17:254-62.

8. Yan X, Wang X, Li Y, et al. Molecular basis for ubiquitin ligase CRL2FEM1C-mediated recognition of C-degron. Nat Chem Biol 2021;17:263-71.

9. Dankert JF, Pagan JK, Starostina NG, et al. FEM1 proteins are ancient regulators of SLBP degradation. Cell Cycle 2017;16:556-64.

10. Wiśniewski JR, Zougman A, Nagaraj N, et al. Universal sample preparation method for proteome analysis. Nat Methods 2009;6:359-62.

11. Zhu Y, Xu H, Chen H, et al. Proteomic analysis of solid pseudopapillary tumor of the pancreas reveals dysfunction of the endoplasmic reticulum protein processing pathway. Mol Cell Proteomics 2014;13:2593-603.

12. Ventura-Holman T, Lu D, Si X, et al. The Fem1c genes: conserved members of the Fem 1 gene family in vertebrates. Gene 2003;314:133-9.

13. Koren I, Timms RT, Kula T, et al. The Eukaryotic Proteome Is Shaped by E3 Ubiquitin Ligases Targeting C-Terminal Degrons. Cell 2018;173:1622-1635.e14.

14. Wang S, Xia W, Qiu M, et al. Atlas on substrate recognition subunits of CRL2 E3 ligases. Oncotarget 2016;7:46707-16.

15. Fang J, Wang W, Fang J, et al. Epithelial-mesenchymal transition classification of circulating tumor cells in lung and colon cancer patients: potential role in clinical practice. Transl Cancer Res 2020;9:6639-51.

16. Li X, Zhao M, He S. RPE epithelial-mesenchymal

Cite this article as: Huang R, Li J, Fu Y, Deng Y. Downregulation of FEM1C enhances metastasis and proliferation in colorectal cancer. Ann Transl Med 2021;9(17):1391. doi: 10.21037/atm-21-4244 transition plays a critical role in the pathogenesis of proliferative vitreoretinopathy. Ann Transl Med 2020;8:263.

17. Wu Y, Yang X, Chen Z, et al. m6A-induced lncRNA RP11 triggers the dissemination of colorectal cancer cells via upregulation of Zeb1. Mol Cancer 2019;18:87.

18. Besson A, Dowdy SF, Roberts JM. CDK inhibitors: cell cycle regulators and beyond. Dev Cell 2008;14:159-69.

19. Tanaka H, Yamashita T, Asada M, et al. Cytoplasmic p21(Cip1/WAF1) regulates neurite remodeling by inhibiting Rho-kinase activity. J Cell Biol 2002;158:321-9.

20. Fournier AE, Takizawa BT, Strittmatter SM. Rho kinase inhibition enhances axonal regeneration in the injured CNS. J Neurosci 2003;23:1416-23.

21. Kedrin D, Wyckoff J, Sahai E, et al. Imaging tumor cell movement in vivo. Curr Protoc Cell Biol 2007; Chapter 19:Unit 19.7.

22. Coffman JA. Cell cycle development. Dev Cell 2004;6:321-7.

23. Gennaro VJ, Stanek TJ, Peck AR, et al. Control of CCND1 ubiquitylation by the catalytic SAGA subunit USP22 is essential for cell cycle progression through G1 in cancer cells. Proc Natl Acad Sci U S A 2018;115:E9298-307.

24. Zheng J, Xue H, Wang T, et al. miR-21 downregulates the tumor suppressor P12 CDK2AP1 and stimulates cell proliferation and invasion. J Cell Biochem 2011;112:872-80.

25. Yuan Z, Gaba AG, Kent TS, et al. Modulation of CDK2AP1 (p12(DOC-1)) expression in human colorectal cancer. Oncogene 2005;24:3657-68.

(English Language Editor: J. Chapnick) 
Supplementary

Table S1 Sequences of primers and siRNAs

\begin{tabular}{|c|c|}
\hline Names & Sequences (5'-3') \\
\hline \multicolumn{2}{|c|}{ Primers for RT-qPCR } \\
\hline FEM1C-qF & GACAAAGCCCGAGTGACC \\
\hline FEM1C-qR & CGTCCTACTGCTITCCAACA \\
\hline GAPDH-qF & GAAGGTGAAGGTCGGAGTC \\
\hline GAPDH-qR & GAAGATGGTGATGGGATITC \\
\hline FEM1A-qF & GCTACACATAGCAGCCCAGA \\
\hline FEM1A-qR & TCTTCTTGAAGGCATTGGTG \\
\hline FEM1B-qF & AAAGGTGGTACGCTTGCTCT \\
\hline FEM1B-qR & TCAATGACATACCCGTCGAA \\
\hline Cullin2-qF & ACGACAATAAAAGCCGTGGTC \\
\hline Cullin2-qR & GGATAGGCCACACATAAAGCAT \\
\hline \multicolumn{2}{|c|}{ Plasmid construction } \\
\hline shFEM1C\#1f & $\begin{array}{l}\text { CCGGAACAACACGACTTTAACCAATCTCGAG } \\
\text { ATTGGTTAAAGTCGTGTTGTTTITTG }\end{array}$ \\
\hline shFEM1C\#1r & $\begin{array}{l}\text { AATTCAAAAAAACAACACGACTTTAACCAATCT } \\
\text { CGAGATTGGTTAAAGTCGTGTTGTT }\end{array}$ \\
\hline \multicolumn{2}{|l|}{ siRNA } \\
\hline siGFP & GCAGAAGAACGGCAUCAAG dTdT \\
\hline siFEM1C\#1 & CAACACGACUUUAACCAAUdTdT \\
\hline siFEM1C\#2 & GGAGCUACAUUUGUAGACAdTdT \\
\hline siFAM1A & AACGCCUCCAGAUCUUCCGdTdT \\
\hline siFAM1B & GCCUAAUGAUUGCGGCAUAdTdT \\
\hline sicullin\#1 & GAACTGCTTGCTAAGTACTGTdTdT \\
\hline sicullin\#2 & GAGCTAGCATTGGATATGTGGdTdT \\
\hline
\end{tabular}




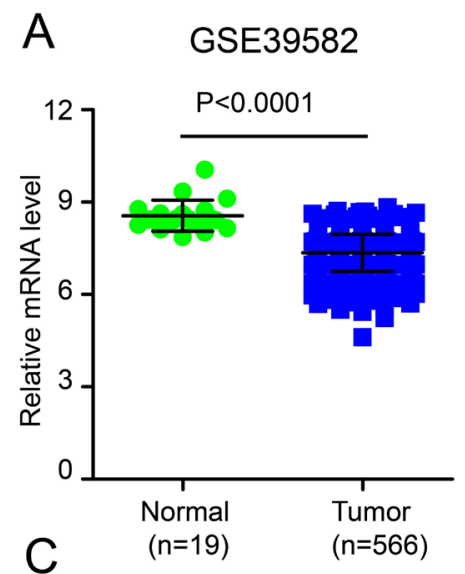

B TCGA
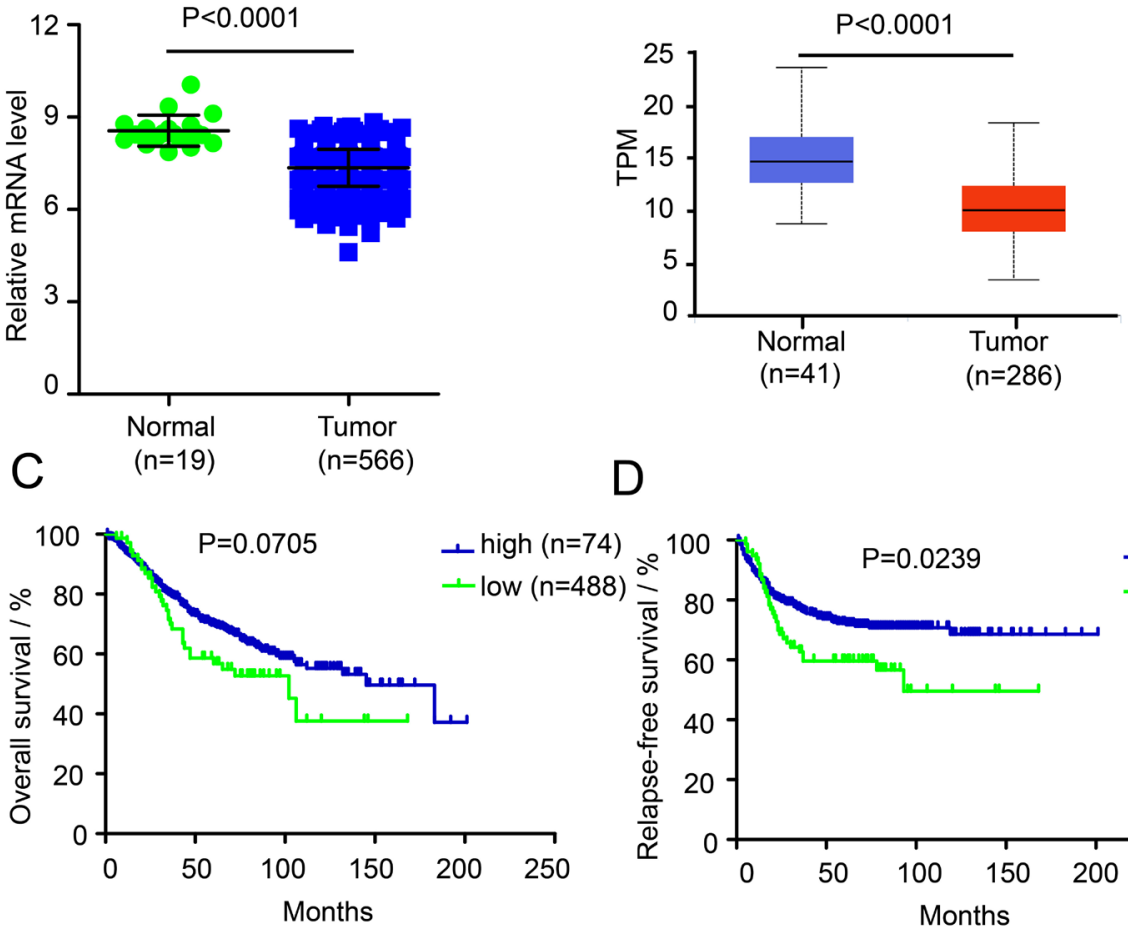

D

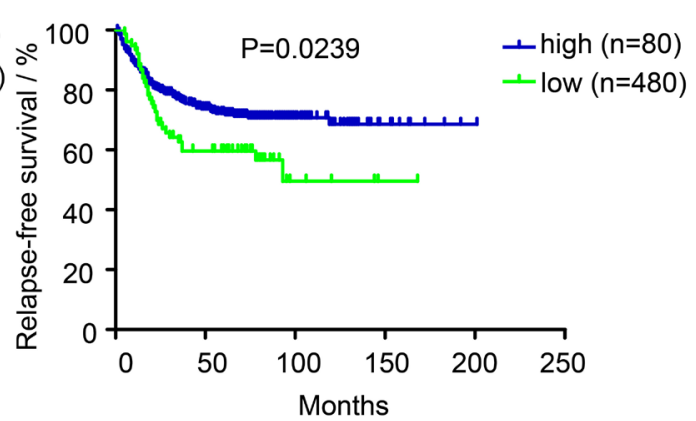

Figure S1 FEM1C is down-regulated in CRC tissue, and its expression level is correlated with prognosis in CRC patients. (A,B) The GEO (A) and TCGA (B) data showed FEM1C mRNA levels in CRC tissues and normal tissues. The TCGA data were downloaded from UALCAN (http://ualcan.path.uab.edu/index.html) (C,D). The relationship between FME1C expression level and survival times (C) or relapse-free survival times (D) in COAD patients was analyzed by the Kaplan-Meier method with a Log-rank test. The data was from GEO (GSE39582). CRC, colorectal cancer. TPM, transcript per million. 

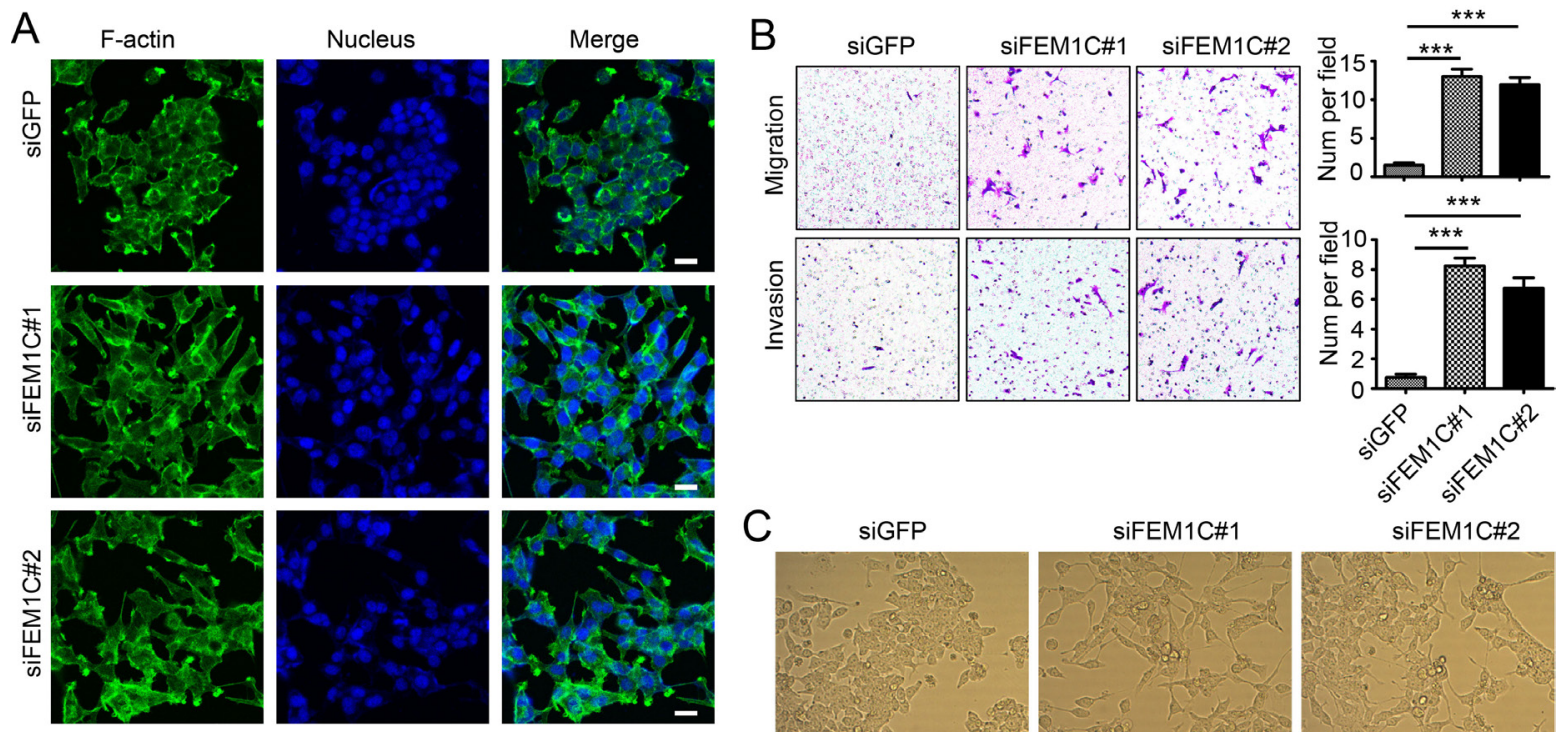

C
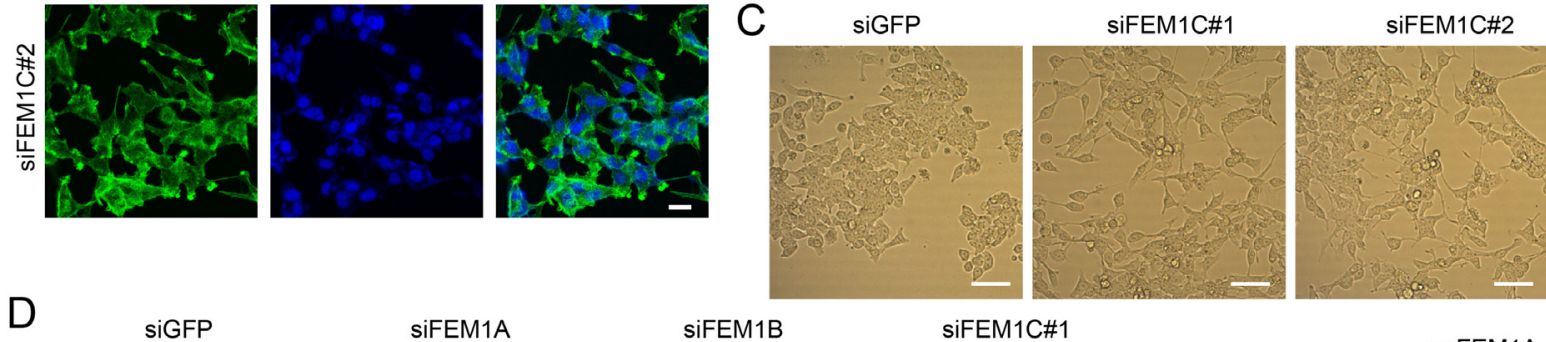

SIFEM1A

SiFEM1B
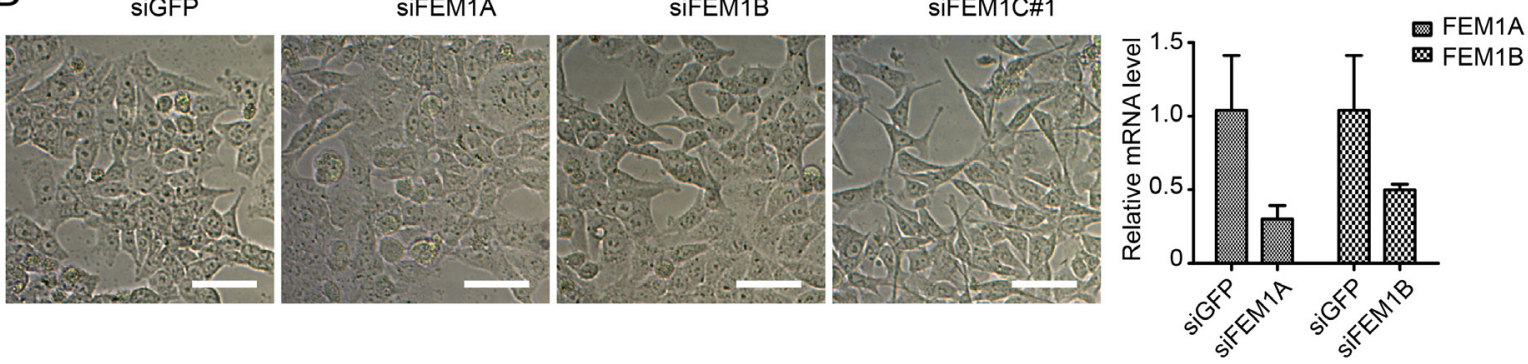

Figure S2 Knockdown of FEM1C resulted in the change of morphology and increased mobility in CRC cells. (A) HCT116 cells were transfected with the indicated siRNAs, and 48 hrs later, F-actin was stained with phalloidin-FITC. Scale bar $=25 \mu \mathrm{m}$. (B) LoVo cells were transfected with indicated siRNAs, and 48 hrs later, the cells were harvested to perform the transwell assay (magnification times, 100x; ***, $\mathrm{P}<0.001$ ). (C) LoVo cells were transfected with indicated siRNAs, and 48 hrs later, the cells were observed on microscopy and photographed. Scale bar $=50 \mu \mathrm{m}$. (D) HCT116 cells were transfected with the indicated siRNAs, and $48 \mathrm{hrs}$ later, the cells were observed in microscopy and photographed (left). The right panel showed the knockdown efficiency of siRNAs. Scale bar $=50 \mu \mathrm{m}$. 

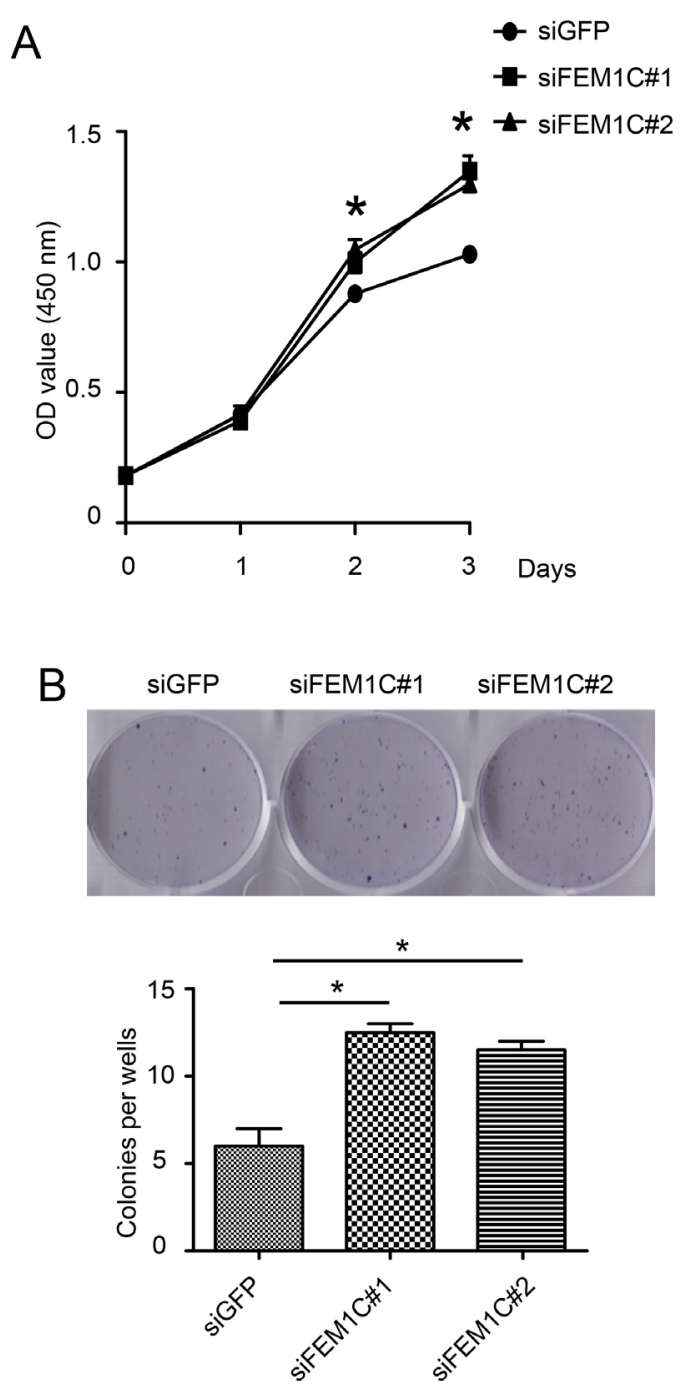

Figure S3 FEM1C knockdown promoted cell proliferation. LoVo cells were transfected with indicated siRNAs, and cell proliferation was determined by the CCK- 8 assay (A) and the colony formation assay (B). *, $\mathrm{P}<0.05$. For colony formation assay, five hundred cells were seeded in 6-well culture plates and cultured for 10 days. Cells were fixed with $4 \%$ paraformaldehyde for $10 \mathrm{~min}$, followed by staining with $1 \%$ crystal violet for $15 \mathrm{~min}$. The plates were photographed, and the colonies were counted.
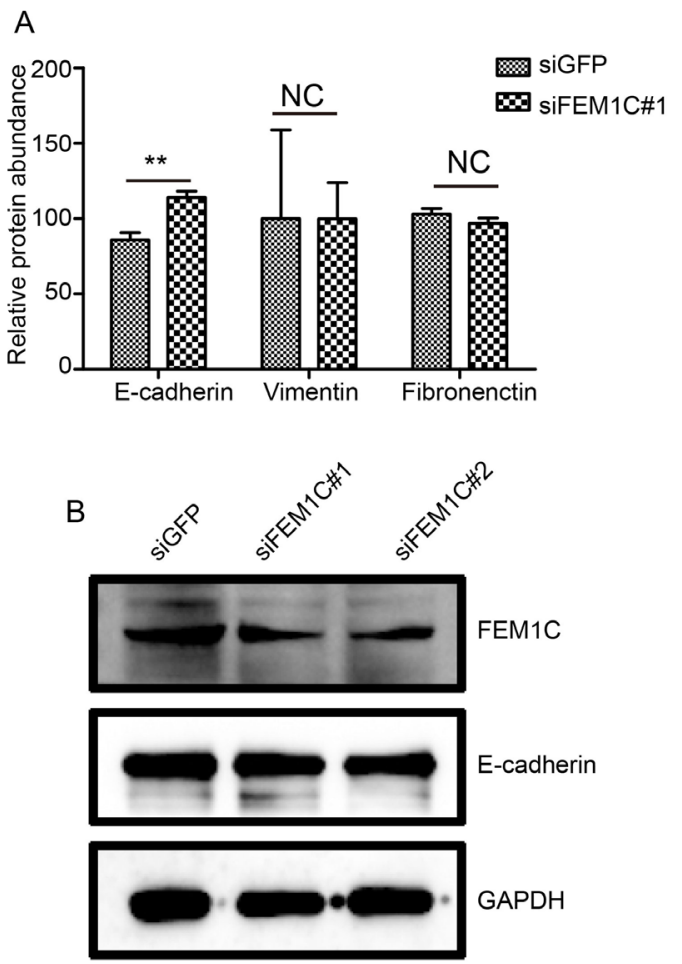

Figure S4 Knockdown of FEM1C did not change the protein level of conventional EMT markers, including vimentin, fibronectin and E-cadherin. (A) Change in the protein level of EMT markers (E-cadherin, vimentin, and fibronectin) after FEM1C knockdown in HCT116 cells. Data were from TMT proteomics analysis between FEM1C knockdown groups and negative control groups (**, $\mathrm{P}<0.01)$. (B) HCT116 cells were transfected with indicated siRNAs, and 48 hrs later, Western Blotting determined the level of the E-cadherin protein. EMT, epithelial-mesenchymal transition. 